In press, Behavioral and Brain Sciences.

\title{
Negative results are needed to show the specific value of a cultural explanation for $g$ : A commentary on Burkart et al.
}

\author{
Arslan, Ruben C. ${ }^{1,2}$ \\ von Borell, Christoph J., ${ }^{1,2}$ \\ Ostner, Julia ${ }^{1,2,3}$ \\ Penke, Lars ${ }^{1,2}$ \\ ${ }^{1}$ Georg August University Göttingen \\ ${ }^{2}$ Leibniz ScienceCampus Primate Cognition \\ ${ }^{3}$ German Primate Center Leibniz Institute for Primate Research
}

\section{Abstract}

The authors suggest that social learning can explain the cognitive positive manifold for social animals including humans. We caution that simpler explanations of positive trait intercorrelations exist, such as genetic load. To test the suggested explanation's specificity, we also need to examine non-social species and traits such as health that are distal to cognitive abilities.

\section{Commentary}

We commend the authors for writing a clear review of the available data on intra-species $g$ and inter-species $G$. Although data on individual differences on non-human animals are scarce, mapping out the potentially far-ranging implications will hopefully encourage more high-quality non-human individual differences research. Their effort to establish clearer and cross-species terms in the modularity debate and to highlight the existence of secondary modules are very welcome. Together with the acknowledgement, as nicely laid out by the authors, that evolution is a tinkerer, not an engineer (Jacob, 1977) we think these efforts will lead to progress in the understanding of the evolution and genetics of general intelligence.

We agree that integration between sub-disciplines currently focused either on universals or individual differences (e.g. evolutionary psychology and behavior genetics) is necessary (Arslan \& Penke, 2015; Penke, Denissen, \& Miller, 2007) and hope warnings about the lack of generalizability in non-human animal cognition research will be heeded (Arden, Bensky, \& Adams, 2016; Thornton \& Lukas, 2012). On the other hand, we are unsure whether the effort to connect the appearance of psychometric $g$ to the presence of general intelligence in a species succeeds. This leads us to examine what could cause $g$ in the absence of a core intelligence trait.

We agree with the authors that psychometric $g$ and domain-general intelligence should not be thoughtlessly equated (Penke et al., 2011). While a species whose individuals vary in a core domain- 
general intelligence ability should exhibit a psychometric $g$, finding a psychometric $g$ does not imply that variation in a core ability causes it. If $g$ were caused by a core ability, then training this ability should show transfer effects to distal cognitive abilities. The authors cite such training studies, but acknowledge controversy about bias and methods (see also Colom et al., 2013; Redick, 2015). Noack, Lövdén, and Schmiedek (2014) conclude the existing literature cannot establish such latent transfer effects. Claims of bilingual advantage have been similarly contested (Paap, Johnson, \& Sawi, 2015). If training of purported core abilities such as executive functions does not increase latent $g$, the case for core abilities causing the positive manifold weakens considerably. Moreover, positive correlations have not only been found among cognitive abilities but also between cognitive abilities and other fitness-related traits such as health, psychopathology and height (Arden et al., 2016). Hagenaars et al. (2016) showed molecular evidence that a shared genetic aetiology underlies the phenotypic associations between health and intelligence.

Reasonable, less cognition-specific explanations have been put forward to explain such positive manifolds. First among them is probably genetic load (Hill et al., in press; Penke et al., 2007). Individuals vary in the number of deleterious genetic mutations they carry. Depending on where they occur, such wrong turns on the genotype-phenotype map could affect the integrity and condition of the whole organism, its brain, or more specific abilities. If many of the variants affecting cognitive abilities are pleiotropic (be that because they affect early development steps or because they disrupt frequently re-used genetic patterns), then we would also expect positive correlations between cognitive abilities and other fitness-related individual differences (Deary, 2012; Houle, 2000). Although causal inference from genetic correlations is hard (Johnson, Penke, \& Spinath, 2011; Solovieff, Cotsapas, Lee, Purcell, \& Smoller, 2013), we ought to consider the possibility that biological pleiotropy, not just health behavior, explains associations between intelligence and health. For example, evolutionarily conserved genomic regions are strongly enriched for genetic variants affecting intelligence (Hill et al., in press). We think this can explain some of the $g$ phenomenon. An explanation based on genetic load can even explain correlations between abilities resulting from primary modules. Other sources of individual differences such as stochastic events in early development can take an explanatory role similar to genetic load, by affecting early developmental steps and pervasive building blocks of the organism (Deary, 2012).

Hence, our null hypothesis should not be complete independence of cognitive abilities, even if we knew they were primary modules. Some intercorrelation should be expected. The expected degree of intercorrelation depends on many unknowns, among them the degree of pleiotropy, the mutational target size, metabolic costs and ontogeny of cognitive abilities.

With the non-human data available so far, we see a gap in the authors' case: the absence of clear negative results in the search for $g / G$. The authors report no taxon where the search for the $g$ factor was conducted with sufficient power and appropriate methods but still failed. We suggest that only after also gathering data from less social species can we ask whether e.g. social learning increases correlations between cognitive abilities. And only when correlations with non-cognitive abilities are compared can we ask what explains the increased correlation between cognitive abilities. As the authors point out, non-human research can help test explanations for $g$ with designs infeasible in humans, such as cross-fostering experiments. This extends to genetic load. Both using genetically uniform strains and mutation accumulation lines (although these are already extremely timeconsuming in micro-organisms) could help clarify the involvement of genetic load. 
We lack the space to fully address alternative explanations for interspecies $G$, but hope to also see joint phylogenetic tree analyses of sociality and variance explained by $g$. To be able to test this, recommendations for increased sample sizes in such studies should be followed (Thornton \& Lukas, 2012). Differential measurement error across subtests and species has to be modelled and corrected for, not just used to explain negative findings.

In conclusion, we would add the following to the authors' call for research: we need individual differences data along the whole gradient of sociality, including even e.g. octopuses, and studies should also examine more distal traits such as health and size. Then, the specific added value of the proposed model can be tested. We want to echo this and previous calls (Arden et al., 2016; Thornton \& Lukas, 2012) for more individual differences research on non-human animal cognition. We hope for more stimulating evolutionary theorizing on individual differences, as in this target article.

\section{References}

Arden, R., Bensky, M. K., \& Adams, M. J. (2016). A Review of Cognitive Abilities in Dogs, 1911

Through 2016: More Individual Differences, Please! Current Directions in Psychological Science, 25, 307-312. doi:10.1177/0963721416667718

Arslan, R. C., \& Penke, L. (2015). Evolutionary genetics. In D. M. Buss (Ed.), Handbook of Evolutionary Psychology (Vol. 2). New York: Wiley.

Colom, R., Román, F. J., Abad, F. J., Shih, P. C., Privado, J., Froufe, M., ... Jaeggi, S. M. (2013). Adaptive n-back training does not improve fluid intelligence at the construct level: Gains on individual tests suggest that training may enhance visuospatial processing. Intelligence, 41, 712-727. doi:10.1016/j.intell.2013.09.002

Deary, I. J. (2012). Looking for "system integrity" in cognitive epidemiology. Gerontology, 58, 545553. doi:10.1159/000341157

Hagenaars, S. P., Harris, S. E., Davies, G., Hill, W. D., Liewald, D. C. M., Ritchie, S. J., ... Deary, I. J. (2016). Shared genetic aetiology between cognitive functions and physical and mental health in UK Biobank ( $\mathrm{N}=112151$ ) and 24 GWAS consortia. Molecular Psychiatry. doi:10.1038/mp.2015.225

Hill, W. D., Davies, G., Harris, S. E., Hagenaars, S. P., The neuroCHARGE Cognitive Working group, Liewald, D., ... Deary, I. (in press). Molecular genetic aetiology of general cognitive function is enriched in evolutionarily conserved regions. Translational Psychiatry. 
Houle, D. (2000). Is there a g factor for fitness? In G. Bock, J. Goode, \& K. Webb (Eds.), The Nature of Intelligence (novartis Foundation Symposium 233) (Vol. 233, pp. 149-170). New York: Wiley. Jacob, F. (1977). Evolution and tinkering. Science, 196, 1161-1166. doi:10.1126/science.860134 Johnson, W., Penke, L., \& Spinath, F. M. (2011). Heritability in the era of molecular genetics: Some thoughts for understanding genetic influences on behavioural traits. European Journal of Personality, 25, 254-266. doi:10.1002/per.836

Noack, H., Lövdén, M., \& Schmiedek, F. (2014). On the validity and generality of transfer effects in cognitive training research. Psychological Research, 78, 773-789. doi:10.1007/s00426-0140564-6

Paap, K. R., Johnson, H. A., \& Sawi, O. (2015). Bilingual advantages in executive functioning either do not exist or are restricted to very specific and undetermined circumstances. Cortex, 69, 265278. doi:10.1016/j.cortex.2015.04.014

Penke, L., Borsboom, D., Johnson, W., Kievit, R. A., Ploeger, A., \& Wicherts, J. M. (2011). Evolutionary psychology and intelligence research cannot be integrated the way Kanazawa (2010) suggested. American Psychologist, 66, 916-917. doi:10.1037/a0024626

Penke, L., Denissen, J. J. A., \& Miller, G. F. (2007). The evolutionary genetics of personality. European Journal of Personality, 21, 549-587. doi:10.1002/per.629

Redick, T. S. (2015). Working memory training and interpreting interactions in intelligence interventions. Intelligence, 50, 14-20. doi:10.1016/j.intell.2015.01.014

Solovieff, N., Cotsapas, C., Lee, P. H., Purcell, S. M., \& Smoller, J. W. (2013). Pleiotropy in complex traits: challenges and strategies. Nature Reviews Genetics, 14, 483-495. doi:10.1038/nrg3461

Thornton, A., \& Lukas, D. (2012). Individual variation in cognitive performance: developmental and evolutionary perspectives. Philosophical Transactions of the Royal Society of London B: Biological Sciences, 367, 2773-2783. doi:10.1098/rstb.2012.0214 